\title{
Fibromyalgieschmerz wegtrainieren
}

Die Fibromyalgie geht mit Gelenk- und Muskelschmerzen einher, ohne dass sich morphologische Veränderungen an Gelenken oder Muskulatur oder pathologische Laborwerte nachweisen lassen. Die Therapie erfolgt multidisziplinär durch Verhaltenstherapie, Biofeedback, Ausdauertraining und Krafttraining. Medikamente sind nur marginal wirksam. Sowohl für Krafttraining wie für aerobes Ausdauertraining ist eine Wirksamkeit auf die Schmerzintensität nachgewiesen. Allerdings gab es bisher keine prospektive randomisierte Vergleichsstudie beider Therapieansätze.

- An der Mayo-Clinic in Rochester wurden 72 FibromyalgiePatienten für eine Studie rekrutiert. Die Hälfte absolvierte ein dreiwöchiges Krafttraining und die andere Hälfte ein aerobes Ausdauertraining. Die Therapie umfasste unter anderem Entspannungsverfahren, Biofeedback, Stressmanagement und Schmerzbewältigung. Der primäre Endpunkt war die Schmerzintensität zu Beginn und am Ende der dreiwöchigen Therapiephase. Sekundäre Endpunkte waren Kraftmessungen in den Beinen und die Schmerzschwellen von Triggerpunkten.

Die initiale Schmerzintensität in der Therapiegruppe mit Krafttraining betrug 46 Einheiten und in der Gruppe mit aerobem Ausdauertraining 48 Punkte, nach drei Wochen betrugen die entsprechenden Schmerzintensitäten 34 und 37 Punkte. Die Unterschiede zwischen den beiden Therapiegruppen waren nicht signifikant. Dies galt auch für alle sekundären Endpunkte.Bei Patientinnen mit Fibromyalgie sind also Krafttraining und aerobes Ausdauertraining gleicherweise wirksam, um eine signifikante Reduktion der Schmerzintensität zu erreichen.

\section{Kommentar}

Diese randomisierte Studie zeigt überzeugend, dass sowohl Krafttraining als auch aerobes Ausdauertraining die Schmerzintensität bei Patientinnen mit Fibromyalgie reduziert. Hier muss allerdings berücksichtigt werden, dass die beiden experimentellen Therapien Bestandteil eines komplexen integrierten Therapieprogramms waren und daher nicht allein für die Verbesserung der Schmerzintensität verantwortlich gemacht werden können. Die hier beobachteten Reduktionen der Schmerzintensität sind klinisch relevant und belegen die Wichtigkeit eines interdisziplinären Therapieansatzes mit körperlicher Ertüchtigung und verhaltenspsychologischen Ansätzen der Schmerztherapie.

Leider kann die Studie nicht die Frage beantworten, ob die Kombination aus Krafttraining und Ausdauersport zu einer weiteren Reduktion der Schmerzintensität führen würde.

- W. M. Hooten et al.

Effects of strength vs aerobic exercise on pain severity in adults with fibromyalgia: A randomized equivalence trial. Pain 153 (2012) 915-923

\section{Hier muss der Dummy durch eine Anzeige ersetzt werden !!}

